\title{
Fundamental Study on Non-invasive Frequency Injection Attack against RO-based TRNG
}

\author{
Saki Osuka ${ }^{1}$, Daisuke Fujimoto ${ }^{1}$, Yu-ichi Hayashi ${ }^{1}$, Naofumi Homma ${ }^{2}$, Arthur Beckers ${ }^{3}$, Joseph Balasch ${ }^{3}$, Benedikt Gierlichs ${ }^{3}$ and \\ Ingrid Verbauwhede ${ }^{3}$ \\ ${ }^{1}$ Nara Institute of Science and Technology, 8916-5 Takayama-cho, Ikoma, Nara 630-0192, Japan \\ 2 Tohoku University, Aramaki Aza Aoba6-3, Aobaku, Sendai 980-8578, Japan \\ ${ }^{3}$ ESAT/COSIC, KU Leuven, Kasteelpark 10, B-3001 Heverlee, Belgium
}

Email: osuka.saki.ok1@is.naist.jp

\begin{abstract}
In this study, we investigate the security threat of non-invasively degrading the randomness of true random number generators (TRNGs) by injecting disturbance waves and estimating the internal state of ring oscillators (ROs) using sidechannel information from outside the device. In addition, we discuss countermeasures against this type of threat.
\end{abstract}

\section{INTRODUCTION}

As a physical attack method that degrades the randomness of the ring oscillator ( $\mathrm{RO}$ )-based true random number generators (TRNGs), frequency injection attacks that require direct access for modifying or invading devices have been proposed [1]. In this study, we investigate the possibility of a remote non-invasive frequency injection attack in the case when close physical access to the TRNGs is difficult to realize.

\section{Method of Non-INVAsive FreQuency InJECTION AtTACK}

A frequency injection attack reduces the fluctuation of transition timing of ROs in TRNGs by injecting sinusoidal waves at certain frequencies thereby locking the ROs (Fig. 1), and degrading the randomness of TRNGs. There are two requirements for the success of non-invasive attacks from a distance: (1) to inject attack signals from outside the device; (2) to estimate that the randomness of the TRNG is impaired from outside the device. For (1), by transmitting sinusoidal waves at certain frequencies via cable, the electric potential of $\mathrm{V}_{\mathrm{dd}} / \mathrm{GND}$ inside the device can be fluctuated [2], and then, attack signals can be injected to the ROs. For (2), we observe the common-mode currents that are caused by the internal processing of the devices, which are then conducted and radiated to the peripheral circuits, to note the changes in the ROs' internal states due to the aforementioned attack.

\section{RESULTS AND DISCUSSION}

Fig. 2 shows our experimental setup. Sinusoidal waves generated by a signal generator are induced by an injection probe into a power cable attached to a TRNG; we observe the common-mode current using an oscilloscope and a current probe clamped to the power cable.

Fig. 3 shows the voltage waveforms between $V_{d d}$ and GND for an RO and the frequency spectrums of the commonmode current generated against time change during the injection of sinusoidal waves. In Fig. 3(a), the RO is not locked, whereas, in Fig. 3(b), the fluctuation of transition timing reduces, and the RO is locked. These results indicate that non-invasive frequency injection attacks via power cable is possible. In addition, we can confirm the changes in the frequency spectrums of the common-mode current based on the operation of ROs. Consequently, it is considered that changes in RO behavior caused by attacks can be observed

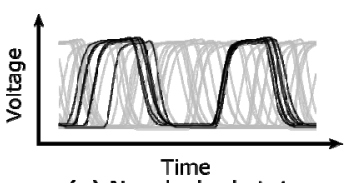

(a) Non-locked state

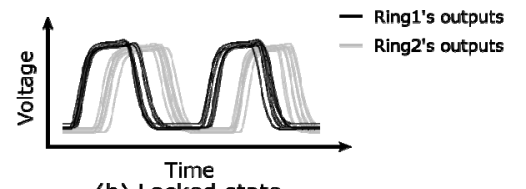

(b) Locked state
Fig. 1 RO waveforms under normal/locked state

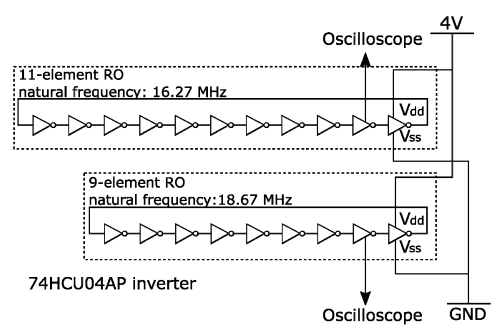

(a) Schematic of RO-based TRNG

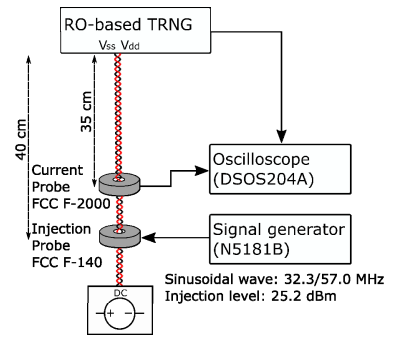

(b) Block diagram of experimental setup
Fig. 2 Experimental setup

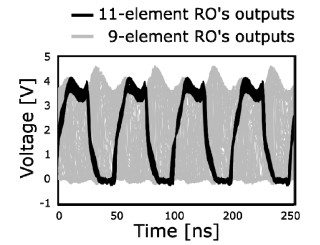

(a) $32.3 \mathrm{MHz}$ injection (Non-locked state)
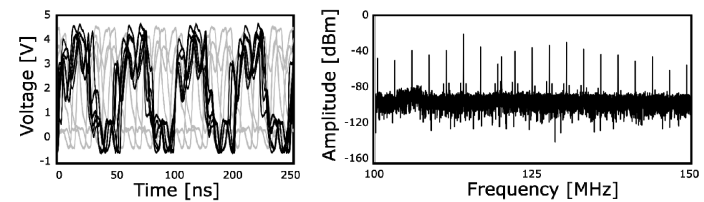

(b) $57.0 \mathrm{MHz}$ injection (Locked state)

Fig. 3 Changes in outputs and frequency spectrums of RO's common-mode currents when under attack

from outside the device. However, because this frequency injection attack is effective only at specific frequencies, one countermeasure could be the suppression of the specific frequencies capable of locking the ROs. For example, by attaching ferrite cores to the power line, it is possible to suppress the frequency bands that could be used for such attacks.

\section{REFERENCES}

[1] A.T. Markettos, S.W. Moore, "The frequency injection attack on ring-oscillator-based true random number generators," CHES, vol.5747, pp.317-331, 2009.

[2] Y. Hayashi, et al. "Transient IEMI threats for cryptographic devices." IEEE Trans. on EMC 55.1 (2013): 140-148. 